\title{
NUMERICAL MODELLING OF SHLIOMIS MODEL BASED FERROFLUID LUBRICATION PERFORMANCE IN ROUGH SHORT BEARING
}

\author{
Mohmmadraiyan M. Munshi \\ Alpha College of Engineering and Technology, Gujarat Technological University, Kalol, Gujarat, India \\ e-mail: raiyan.munshi@gmail.com
}

Ashok R. PATEL

Vishwakarma Government Engineering College, Gujarat Technological University, Ahmedabad, Gujarat, India e-mail:dr.arpatel69@gmail.com

Gunamani Deheri

Department of Mathematics, Sardar Patel University, Vallabh Vidyanagar, Gujarat, India

e-mail: gm.deheri@rediffmail.com

\begin{abstract}
This paper has attempted to scrutinize the bearing performance of a rough short bearing assisted by a ferrofluid with the help of numerical modelling of the Shliomis model. The transverse roughness is calculated stochastically by averaging the Christensen and Tonder models. A non-zero mean is assumed for the probability density function for the random variable that determines the roughness of the bearing which is symmetrical. This attempt is made to create a more pragmatic and applicable situation. Expressions that can signify a dimensionless form of pressure and bearing load carrying capacity are found using Reynolds' equation. The load carrying capacity equation is then solved numerically with the help of Simpson's 1/3 rule to analyze the impact on the bearing system. From the graphical representation, it can be concluded that ferrofluid lubrication based on the Shliomis model can significantly neutralize the negative effects of the bearing roughness on its load carrying capacity.
\end{abstract}

Keywords: ferrofluid, roughness, load carrying capacity

\section{Introduction}

The slider bearing is one of the most basic and commonly used hydrodynamic bearing. The simplicity of the film thickness expression and the straightforwardness of boundary conditions can be held accountable for the same. Unlike other bearings, slider bearings do not create negative pressure which can be problematic for load bearing. This is because their film is continuous and non-diverging. Thus, they support axial loads. Many researchers have studied non-porous sliders. Christensen and Tonder (1969a,b, 1970) used a stochastic concept and came up with a new model for lubricated surfaces with striated roughness using an averaging film. They derived stochastic Reynolds' equation and used the results to study the impact of surface roughness on the load bearing capacity in a rough bearing system. Shliomis (1974) analyzed the modes of creating magnetic colloids along with the stability concerns. Patel et al. (2010b) worked on studying the performance of a smooth short bearing. Deheri and Patel (2011) and Patel et al. (2010a) analyzed the performance of a short rough bearing with a zero mean. They worked with a variety of magnetic field magnitudes in their study. Shimpi and Deheri (2010) further worked on the results of (Deheri and Patel, 2011; Patel et al., 2010a) focusing on short bearings with the non-zero mean with a different form of the magnetic field magnitude. Shimpi and Deheri (2012) worked on it by including a deformation effect as well. Patel and Deheri (2013a) studied 
further comparing two different types of porous structures. Patel and Deheri (2013b) extended the study of (Patel et al., 2010b) by adding the aspect of slip velocity. They concluded that optimal performance can only be achieved at the minimum slip. It should be noted that the researches mentioned above used the Neuringer-Rosensweig model. Patel et al. (2015) conducted a different study by replacing the hydrodynamic bearing of the above-mentioned studies with a hydromagnetic porous short bearing. Additionally, it is a commonly known fact that roughness impacts the load carrying capacity substantially.

By reviewing literature concerning ferrofluid flow, one can understand that the Shliomis model displays better results than the Neuringer-Rosensweig model. Thus, this study is focused on scrutinizing the performance of ferrofluid lubrication using a short bearing based on the Shliomis model.

\section{Analysis}

The configuration of the short bearing system (infinitely short in the $z$-direction) is presented in Fig. 1. The slider has a velocity $U$ in the $x$-direction. The breadth $B$ is in the $z$-direction where $B \ll L$ (length). The pressure gradient $\partial p / \partial x$ can be neglected as the pressure gradient $\partial p / \partial z$ remains much larger.

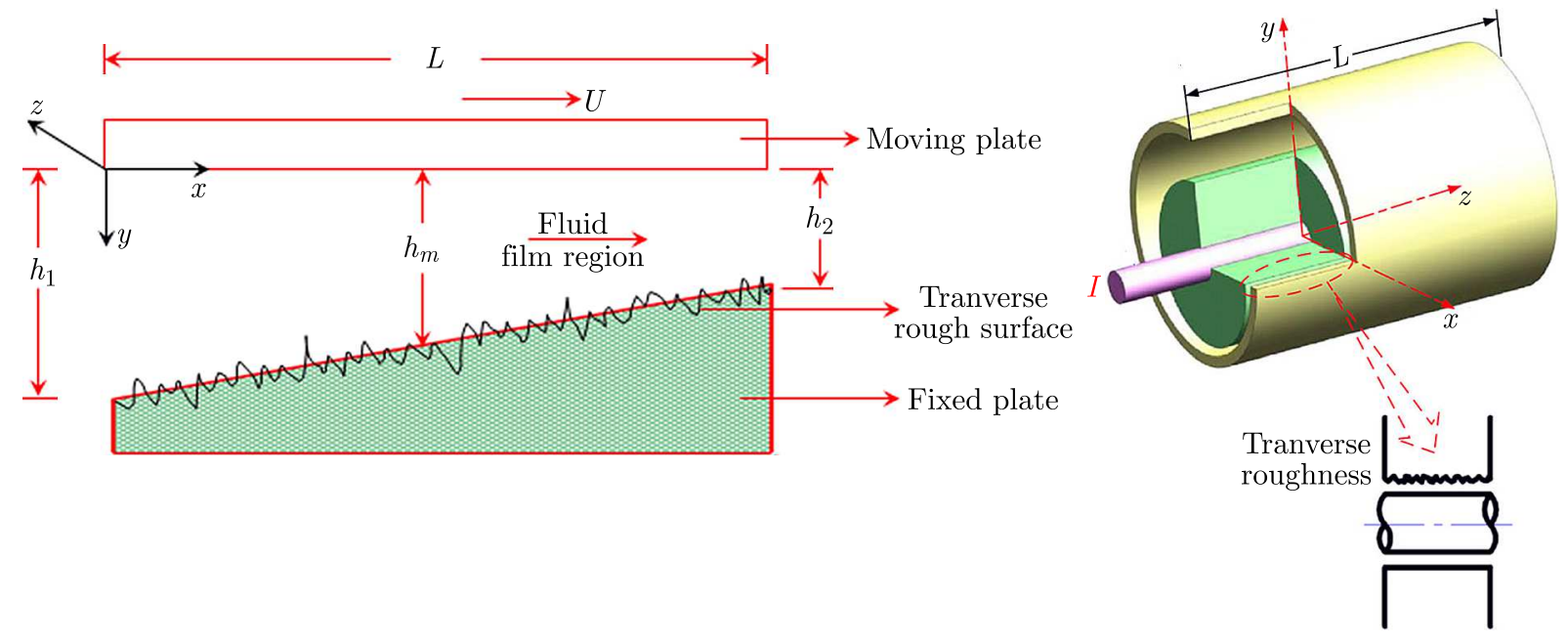

Fig. 1. Configuration of the bearing system (Patel and Deheri, 2013a)

The thickness $h$ is considered as

$$
h=h_{m}+h_{s}
$$

where $h_{m}$ is taken as (Patel and Deheri, 2013a)

$$
h_{m}=h_{2}\left[1+m\left(1-\frac{x}{L}\right)\right] \quad m=\frac{h_{1}-h_{2}}{h_{2}}
$$

using the works of (Christensen and Tonder, 1969a,b, 1970). Also, the study uses $h_{s}$ using the probability density function (Christensen and Tonder, 1969a,b, 1970)

$$
f\left(h_{s}\right)= \begin{cases}\frac{35}{32 c}\left(1-\frac{h_{s}^{2}}{c^{2}}\right)^{3} & -c \leqslant h_{s} \leqslant c \\ 0 & \text { elsewhere }\end{cases}
$$


with $c$ being the maximum deviation from the mean film thickness. $\alpha, \sigma$ and $\varepsilon$ are given by the relationships (Christensen and Tonder, 1969a,b, 1970)

$$
\alpha=E\left(h_{s}\right) \quad \sigma^{2}=E\left(h_{s}-\alpha\right)^{2} \quad \varepsilon=E\left(h_{s}-\alpha\right)^{3}
$$

where the expectancy operator is given by

$$
E(\cdot)=\int_{-c}^{c}(\cdot) f\left(h_{s}\right) d h_{s}
$$

Actually, magnetic fluids or ferrofluids have a constant nature and are a type of colloidal suspensions that possess extremely superior magnetic particles in a viscous fluid. We can use an external magnetic field to position, limit or monitor these fluids as required. This, in turn, increases the fluid effective viscosity. This research has substantially contributed to an increased application of these magnetic fluids in bearing systems as lubricating agents. A noteworthy fact here is that major studies in the field use the Neuringer-Rosensweig model suggesting that magnetization vector is parallel to the applied magnetic field. Since the Shliomis model considers particle rotation, it overcomes this limitation.

Shliomis $(1972,1974)$ proposed that a change in the applied magnetic field can have a two-way implication on the particles in the magnetic fluid. Either, rotation of such particles is impacted or the magnetic moment changes in them. $\tau_{B}$ (Brownian relaxation time parameter) is used to derive particle rotation and $\tau_{S}$ (magnetic moment relaxation time parameter) suggests the intrinsic process of rotation. Considering a steady flow while overlooking the inertial and second derivatives of $\mathbf{S}$, the equations of flow becomes

$$
-\nabla p+\eta \nabla^{2} \mathbf{q}+\mu_{0}(\mathbf{M} \cdot \nabla) \mathbf{H}+\frac{1}{2 \tau_{s}} \nabla \times(\mathbf{S}-I \mathbf{\Omega})=\mathbf{0}
$$

where

$$
\begin{array}{llrl}
\mathbf{\Omega} & =\frac{1}{2} \nabla \times \mathbf{q} & \mathbf{S}=I \boldsymbol{\Omega}+\mu_{0} \tau_{s}(\mathbf{M} \times \mathbf{H}) \\
\mathbf{M}=M_{0} \frac{\mathbf{H}}{H}+\frac{\tau_{B}}{I}(\mathbf{S} \times \mathbf{M}) & \nabla \times \mathbf{H}=\mathbf{0}
\end{array}
$$

and

$$
\nabla(\mathbf{H}+\mathbf{M})=0
$$

The following can be derived by combining all the above mentioned equations; in the light of the procedure given in (Shliomis, 1974)

$$
\begin{aligned}
& -\nabla p+\eta \nabla^{2} \mathbf{q}+\mu_{0}(\mathbf{M} \cdot \nabla) \mathbf{H}+\frac{1}{2} \mu_{0} \nabla \times(\mathbf{M} \times \mathbf{H})=\mathbf{0} \\
& \mathbf{M}=M_{0} \frac{\mathbf{H}}{H}+\tau_{B} \boldsymbol{\Omega} \times \mathbf{M}+\frac{\mu_{0} \tau_{B} \tau_{s}}{I}(\mathbf{M} \times \mathbf{H}) \times \mathbf{M}
\end{aligned}
$$

Langevin's parameter $\xi>1$ is used for a strong magnetic field, the above equation (Shliomis, 1972, 1974; Bhat, 2003) changes to

$$
\mathbf{M}=\frac{M_{0}}{H}\left[\mathbf{H}+\tau_{B}(\boldsymbol{\Omega} \times \mathbf{H})\right]
$$

with

$$
\bar{\tau}=\frac{6 \eta \phi}{n k_{B} T(1+\xi \operatorname{coth} \xi)}
$$


where

$$
M_{0}=n \mu\left(\operatorname{coth} \xi-\frac{1}{\xi}\right) \quad H=\frac{k_{B} T \xi}{\mu_{0} \mu}
$$

For a deferment of spherical particles

$$
\frac{I}{\tau_{s}}=6 \eta \phi \quad \tau_{B}=\frac{3 \eta V}{k_{B} T}
$$

In view of the discussion (Shliomis, 1974), the flow remains in the $x z$-plane while the magnetic field is taken in the $y$-direction by making use of the assumptions for $U=(u, v, w)$ and $\mathbf{H}=\left[H_{x}, H_{y}, H_{z}\right]$ where in $u, w \gg v$ and $H_{y} \gg H_{x}, H_{z}$. In the light of the boundary conditions of the magnetic field componants at the plates, it can be infered that $H_{y} \approx H_{0}$ while $H_{x}, H_{z}$ remain negligible in comparison with $H_{0}$. Therefore, for the axially symmetric flow, the associated uniform magnetic field may be represented by $\mathbf{H}=\left[0, H_{0}, 0\right]$.

Equations (2.8) and (2.9) develop into the following (Majumdar, 2008)

$$
\frac{\partial p}{\partial x}=\frac{\partial}{\partial y}\left(\eta(1+\tau) \frac{\partial u}{\partial y}\right) \quad \frac{\partial p}{\partial y}=0 \quad \frac{\partial p}{\partial z}=\frac{\partial}{\partial y}\left(\eta(1+\tau) \frac{\partial w}{\partial y}\right)
$$

and

$$
\frac{\partial \rho}{\partial t}+\frac{\partial}{\partial x}(\rho u)+\frac{\partial}{\partial y}(\rho v)+\frac{\partial}{\partial z}(\rho w)=0
$$

where (referring to Shliomis, 1972)

$$
\tau(\phi, \xi)=\frac{3}{2} \phi \frac{\xi-\tanh \xi}{\xi+\tanh \xi} \quad \eta=\eta_{0}\left(1+\frac{5}{2} \phi\right)
$$

Solving equation (2.13) under no slip boundary conditions (Majumdar, 2008)

$$
\begin{array}{lll}
\text { at } y=0: & u=u_{b} & w=w_{b} \\
\text { at } y=h: & u=u_{a} & w=w_{a}
\end{array}
$$

one can find

$$
\begin{aligned}
& u=\frac{1}{2 \eta(1+\tau)} \frac{\partial p}{\partial x} y(y-h)+\frac{h-y}{h} u_{b}+\frac{y}{h} u_{a} \\
& w=\frac{1}{2 \eta(1+\tau)} \frac{\partial p}{\partial z} y(y-h)+\frac{h-y}{h} w_{b}+\frac{y}{h} w_{a}
\end{aligned}
$$

Putting the velocity components expression into continuity equation (2.14) and integrating under the conditions $v=v_{b}$ at $y=0$ and $v=v_{a}$ at $y=h$ gives rise to

$$
\begin{gathered}
\rho\left(v_{a}-v_{b}\right)=-\frac{1}{2}\left\{\int_{0}^{h} \frac{\partial}{\partial x}\left[\frac{\rho}{\eta(1+\tau)} \frac{\partial p}{\partial x} y(y-h)\right] d y+\int_{0}^{h} \frac{\partial}{\partial z}\left[\frac{\rho}{\eta(1+\tau)} \frac{\partial p}{\partial z} y(y-h)\right] d y\right\} \\
-\int_{0}^{h} \frac{\partial}{\partial x}\left\{\rho\left[\left(\frac{h-y}{h}\right) u_{b}+\frac{y}{h} u_{a}\right]\right\} d y-\int_{0}^{h} \frac{\partial}{\partial z}\left\{\rho\left[\left(\frac{h-y}{h}\right) w_{b}+\frac{y}{h} w_{a}\right]\right\} d y-h \frac{\partial \rho}{\partial t}
\end{gathered}
$$

In reference to the experiment through the utilization of relation (Majumdar, 2008)

$$
\int_{0}^{h} \frac{\partial}{\partial x}[f(x, y, z)] d z=\frac{\partial}{\partial x} \int_{0}^{h} f(x, y, z) d z-f(x, y, h) \frac{\partial h}{\partial x}
$$


one obtains for constant velocities

$$
\frac{\partial}{\partial x}\left(\frac{\rho h^{3}}{12 \eta(1+\tau)} \frac{\partial p}{\partial x}\right)+\frac{\partial}{\partial z}\left(\frac{\rho h^{3}}{12 \eta(1+\tau)} \frac{\partial p}{\partial z}\right)=\frac{U}{2} \frac{\partial}{\partial x}(\rho h)+\frac{\partial}{\partial t}(\rho h)
$$

where

$$
U=u_{a}+u_{b}
$$

Generalized Reynolds' equation (2.19) turns in the state of equilibrium, which brings (Majumdar, 2008)

$$
\frac{\partial}{\partial x}\left(\frac{\rho h^{3}}{12 \eta(1+\tau)} \frac{\partial p}{\partial x}\right)+\frac{\partial}{\partial z}\left(\frac{\rho h^{3}}{12 \eta(1+\tau)} \frac{\partial p}{\partial z}\right)=\frac{U}{2} \frac{\partial}{\partial x}(\rho h)
$$

Modified two dimensional Reynolds' equation for an incompressible lubricant is

$$
\frac{\partial}{\partial x}\left(\frac{h^{3}}{12 \eta(1+\tau)} \frac{\partial p}{\partial x}\right)+\frac{\partial}{\partial z}\left(\frac{h^{3}}{12 \eta(1+\tau)} \frac{\partial p}{\partial z}\right)=\frac{U}{2} \frac{\partial h}{\partial x}
$$

In the $x$-direction, the flow because of pressure gradient in the variation of pressure can be avoided when the bearing is short. In this case, one dimensional equation (2.22) leads to

$$
\frac{\partial}{\partial z}\left(h^{3} \frac{\partial p}{\partial z}\right)=6 \eta(1+\tau) U \frac{\partial h}{\partial x}
$$

which is Reynolds' equation (Basu et al., 2005; Majumdar, 2008; Shimpi and Deheri, 2010) modified according to the general hydrodynamic lubrication assumptions.

According to the stochastically average process (Shimpi and Deheri, 2010), (2.23) becomes

$$
\begin{aligned}
& \frac{\partial}{\partial z}\left(E\left(h^{3}\right) \frac{\partial p}{\partial z}\right)=6 \eta(1+\tau) U \frac{\partial}{\partial x}(E(h)) \\
& \frac{\partial}{\partial z}\left(g\left(h_{m}, \alpha, \sigma, \varepsilon\right) \frac{\partial p}{\partial z}\right)=6 \eta(1+\tau) U \frac{\partial}{\partial x}\left(h_{m}+\alpha\right)
\end{aligned}
$$

where

$$
g\left(h_{m}, \alpha, \sigma, \varepsilon\right)=h_{m}^{3}+3 \alpha h_{m}^{2}+3\left(\sigma^{2}+\alpha^{2}\right) h_{m}+3 \sigma^{2} \alpha+\alpha^{3}+\varepsilon
$$

The following dimensionless quantities are used

$$
\begin{array}{cccc}
\bar{X}=\frac{x}{L} & \bar{Z}=\frac{z}{B} & \bar{h}=\frac{h_{m}}{h_{2}}=1+m(1-\bar{X}) \\
\bar{g}(\bar{h}, \bar{\alpha}, \bar{\sigma}, \bar{\varepsilon})=\frac{g\left(h_{m}, \alpha, \sigma, \varepsilon\right)}{h_{2}^{3}} & \bar{\alpha}=\frac{\alpha}{h_{2}} & \bar{\sigma}=\frac{\sigma}{h_{2}} \\
\bar{\varepsilon}=\frac{\varepsilon}{h_{2}^{3}} & \bar{p}=\frac{p h_{2}^{3}}{\eta_{0} U B^{2}} & \bar{L}=\frac{L}{h_{2}} & \bar{B}=\frac{B}{h_{2}}
\end{array}
$$

The associated boundary conditions (Lin et al., 2013; Patel et al., 2012; Deheri and Patel, 2011) are

$$
\bar{p}=0 \quad \text { at } \quad \bar{Z}= \pm \frac{1}{2} \quad \text { and } \quad \frac{d \bar{p}}{d \bar{Z}}=0 \quad \text { at } \quad \bar{Z}=0
$$

With the aid of (2.27), the pressure distribution in a non-dimensional form comes out to be

$$
\bar{p}=\frac{3 m(1+2.5 \phi)(1+\tau)}{\bar{L} \bar{g}(\bar{h}, \bar{\alpha}, \bar{\sigma}, \bar{\varepsilon})}\left(\frac{1}{4}-\bar{Z}^{2}\right)
$$


where

$$
\bar{g}(\bar{h}, \bar{\alpha}, \bar{\sigma}, \bar{\varepsilon})=\bar{h}^{3}+3 \bar{\alpha} \bar{h}^{2}+3\left(\bar{\sigma}^{2}+\bar{\alpha}^{2}\right) \bar{h}+3 \bar{\sigma}^{2} \bar{\alpha}+\bar{\alpha}^{3}+\bar{\varepsilon}
$$

The load bearing capacity in the dimensionless form (Patel and Deheri, 2013a) is obtained

$$
\bar{W}=\frac{h_{2}^{3} W}{\eta_{0} U B^{4}} \quad \bar{W}=\frac{m(1+2.5 \phi)(1+\tau)}{2 \bar{B}} \int_{0}^{1} \frac{d \bar{X}}{\bar{g}(\bar{h}, \bar{\alpha}, \bar{\sigma}, \bar{\varepsilon})}
$$

where the load bearing capacity is calculated using (Patel and Deheri, 2013a)

$$
W=\int_{-\frac{B}{2}}^{\frac{B}{2}} \int_{0}^{L} p(x, z) d x d z
$$

\section{Results and discussion}

The final values of $\bar{W}$ using different parameters are plotted graphically. Figures 2a-2f display the changes in $\bar{W}$ corresponding to different values of $\tau$. They suggest that magnetization leads

(a)

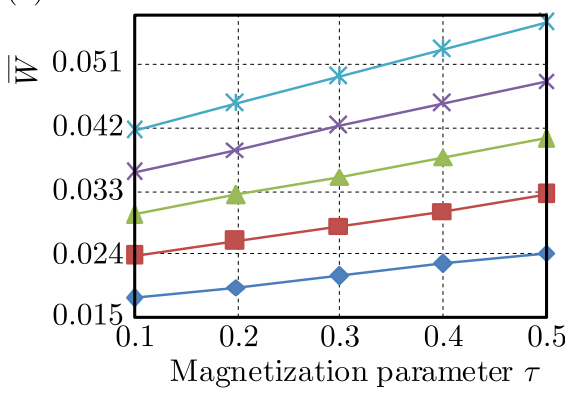

(c)

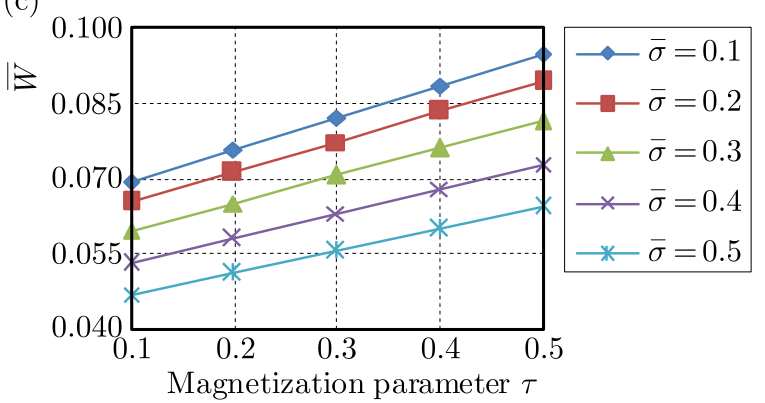

(e)
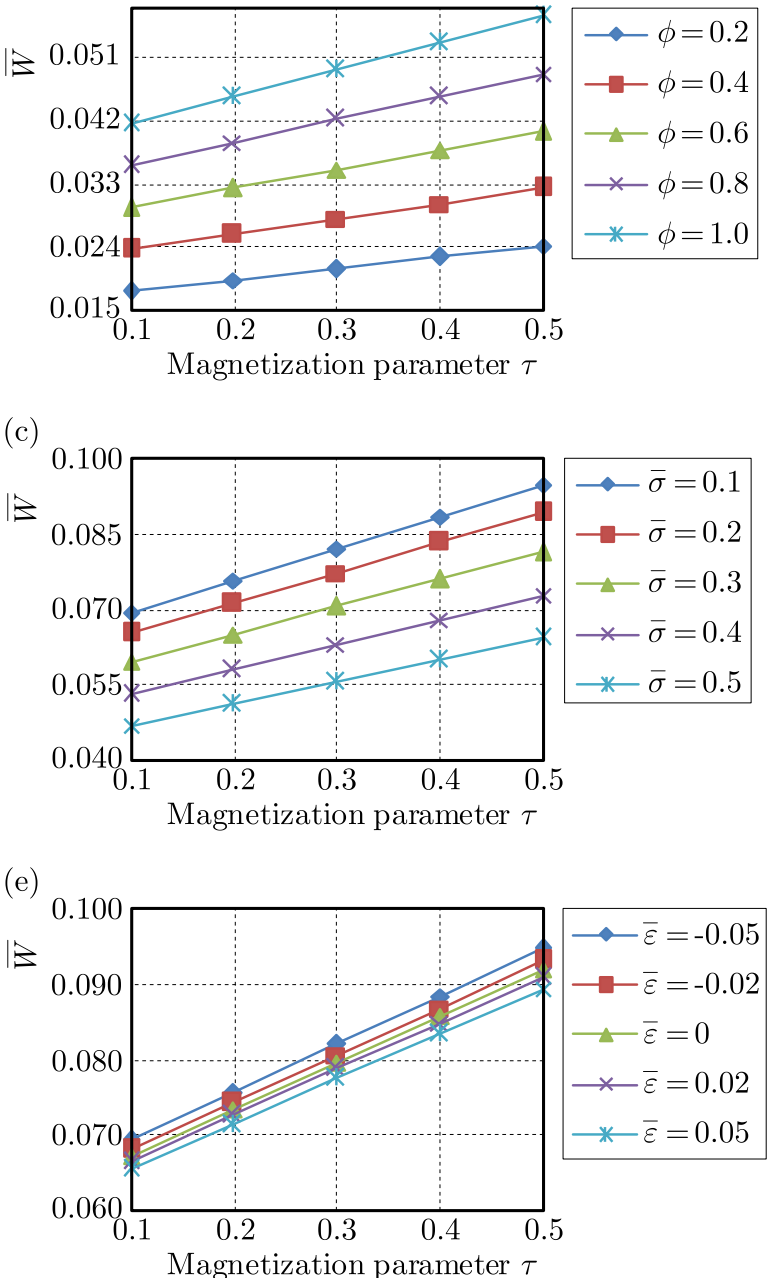

(b)

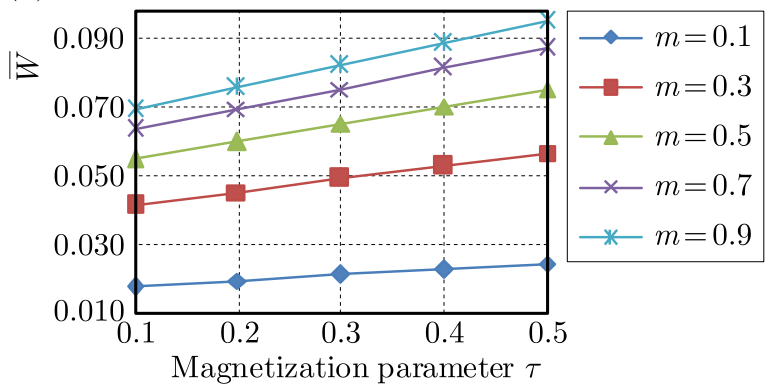

(d)

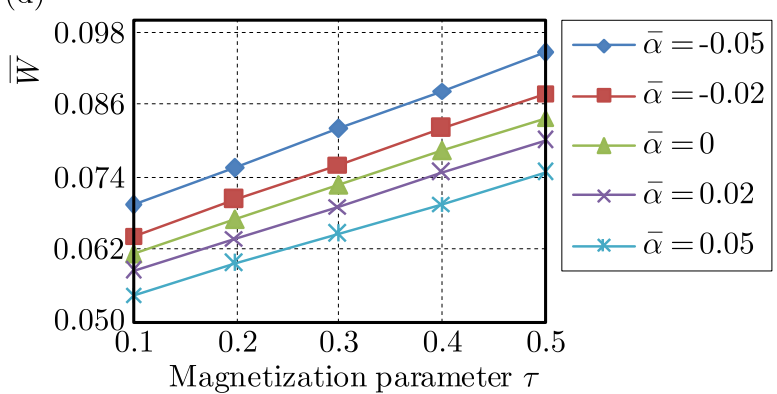

(f)

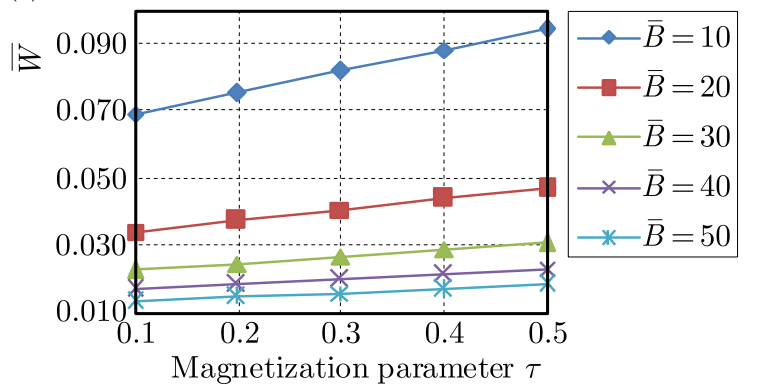

Fig. 2. Trends of $\bar{W}$ concerned with $\tau$ and: (a) $\phi$, (b) $m$, (c) $\bar{\sigma}$, (d) $\bar{\alpha}$, (e) $\bar{\varepsilon}$, (f) $\bar{B}$ 
to a substantial increase in $\bar{W}$. It is noticed from Fig. 2e that the value of maximum $\bar{W}$ derived is 0.094 at a smaller value of $\bar{\varepsilon}=-0.05$ with regards to $\tau$.

From Figs. 3a-3e, it can be suggested that with an increase in $\bar{W}, \phi$ shifts from 0.2 to 1.

(a)

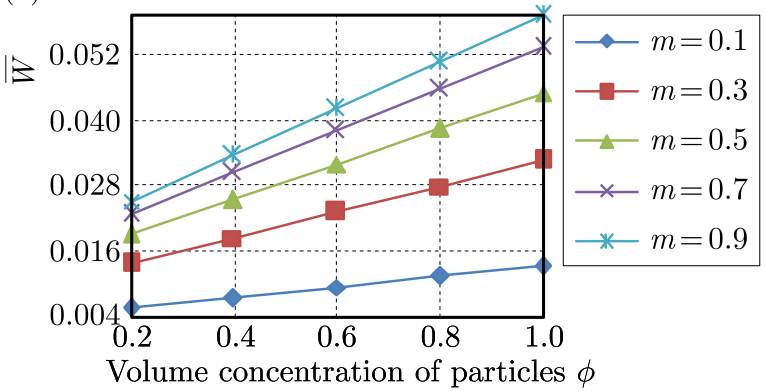

(c)

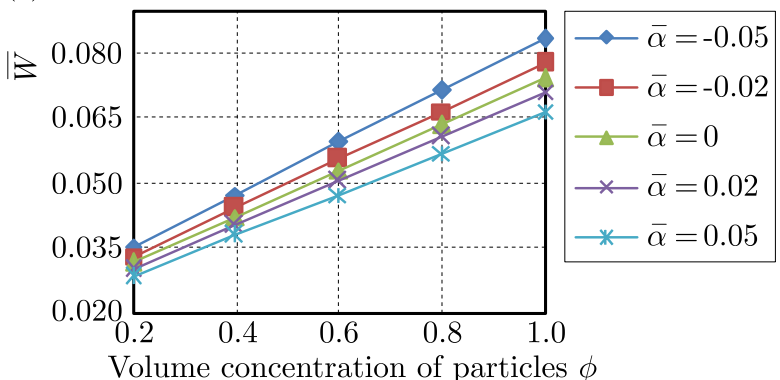

(e)

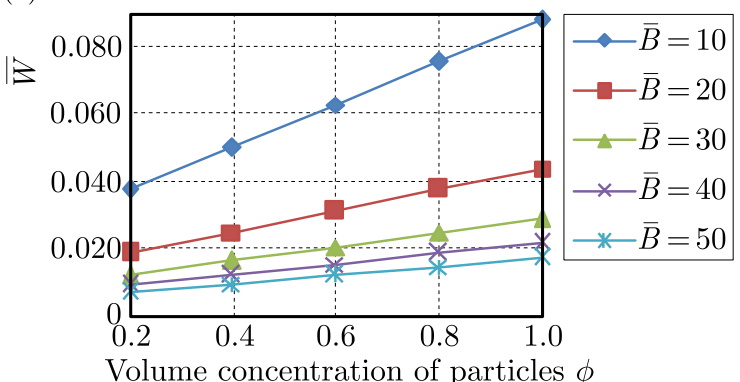

(b)

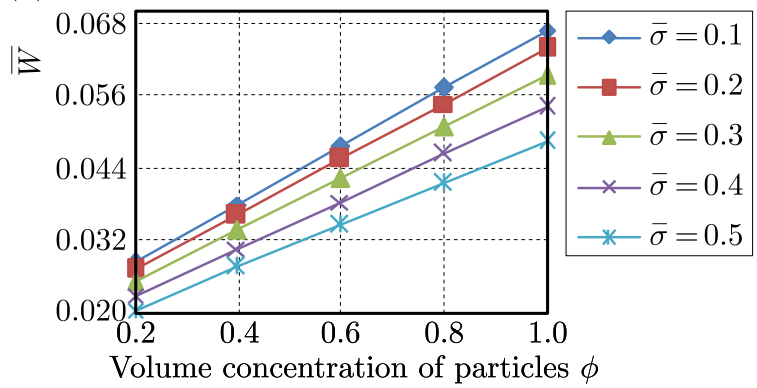

(d)

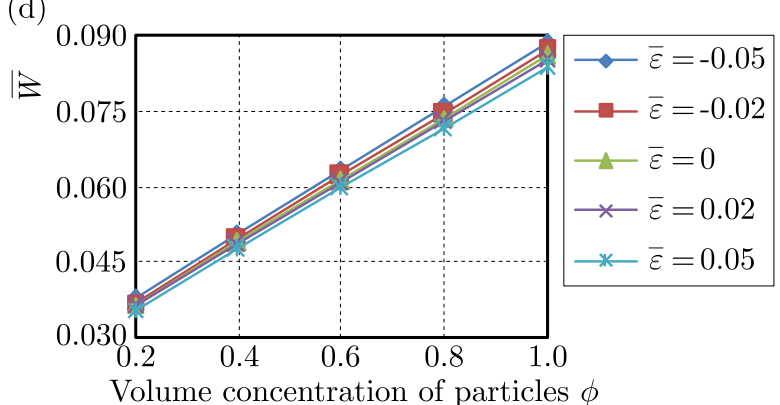

Fig. 3. Trends of $\bar{W}$ concerned with $\phi$ and: (a) $m$, (b) $\bar{\sigma}$, (c) $\bar{\alpha}$, (d) $\bar{\varepsilon}$, (e) $\bar{B}$

Figures $4 \mathrm{a}-4 \mathrm{~d}$ display the impact of the aspect ratio on $\bar{W}$ and suggests that the aspect ratio causes a sharp increase in $\bar{W}$.

The decrease in the value of $\bar{W}$ in Figs. 5a-5c displays the unfavorable impact of standard deviation on the bearing system performance.

Figures $6 \mathrm{a}$ and $6 \mathrm{~b}$ establish an inverse relationship between the variance and $\bar{W}$ suggesting that positive variance reduces $\bar{W}$ while negative variance increases it. Under the impact of skewness, $\bar{W}$ changes according to Fig. 7 . 
(a)

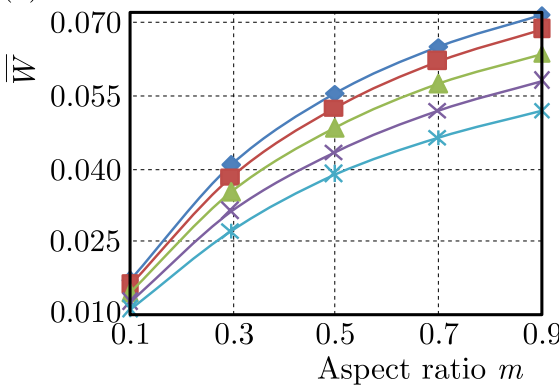

(c)
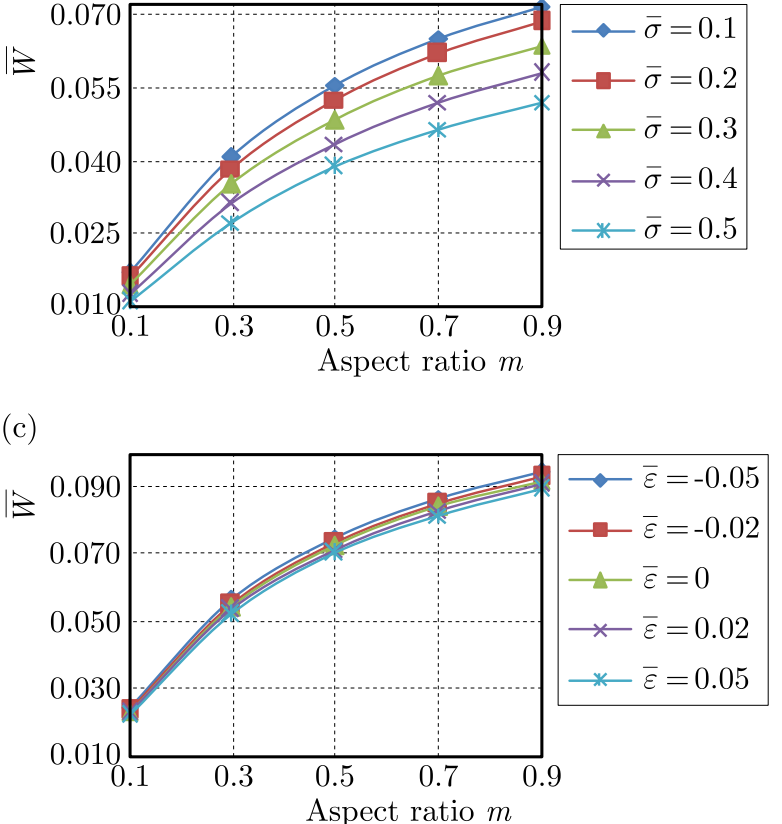

(b)

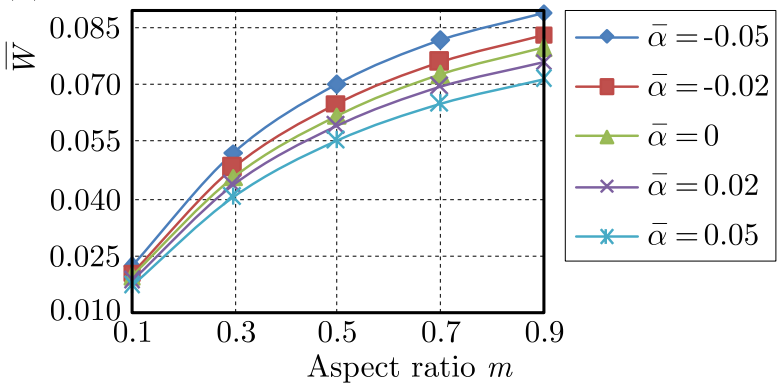

(d)

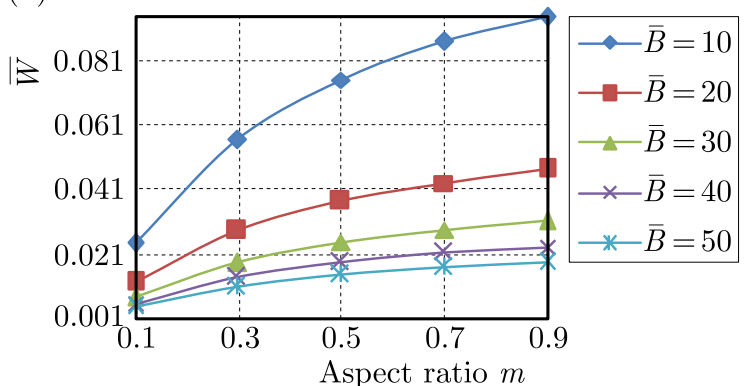

Fig. 4. Trends of $\bar{W}$ concerned with $m$ and: (a) $\bar{\sigma}$, (b) $\bar{\alpha}$, (c) $\bar{\varepsilon}$, (d) $\bar{B}$

(a)

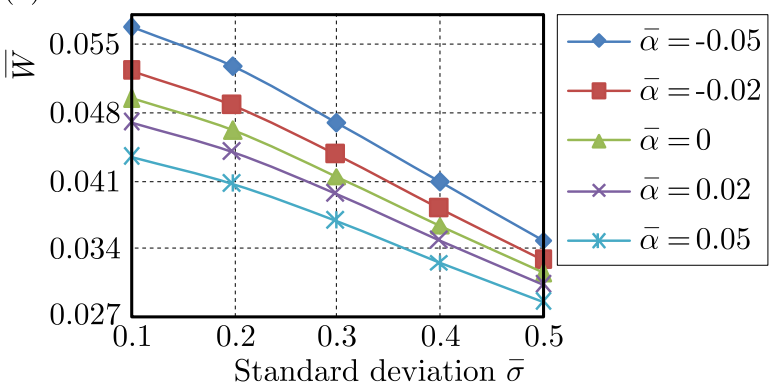

(c)

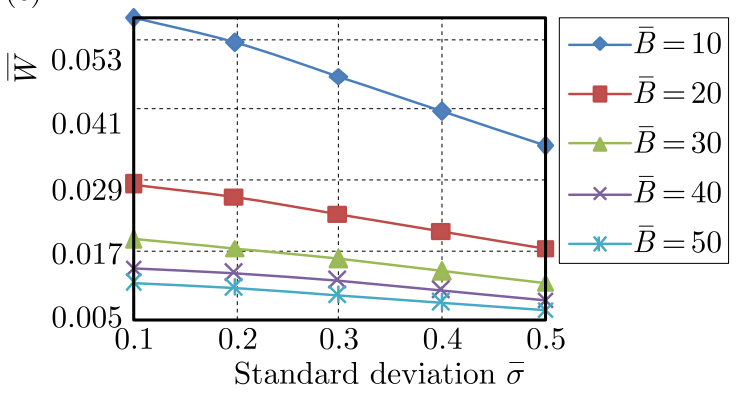

(b)

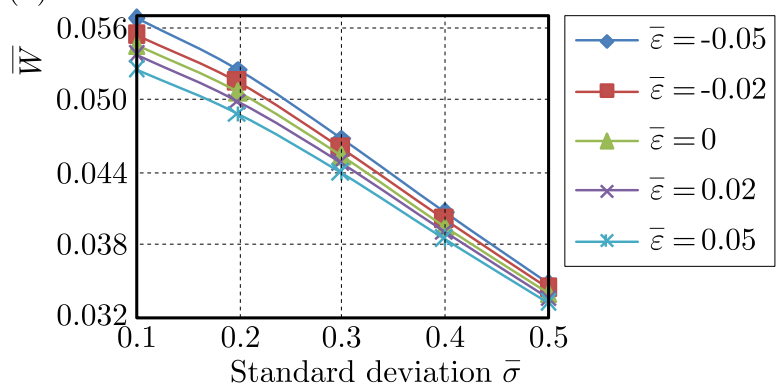

Fig. 5. Trends of $\bar{W}$ concerned with $\bar{\sigma}$ and: (a) $\bar{\alpha}$, (b) $\bar{\varepsilon}$, (c) $\bar{B}$

(a)

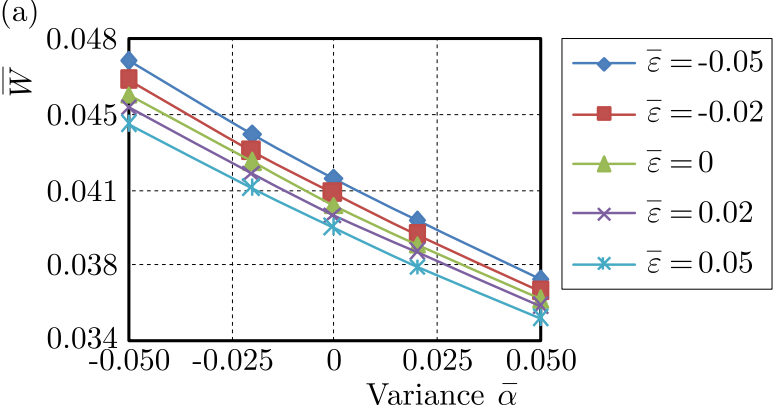

(b)

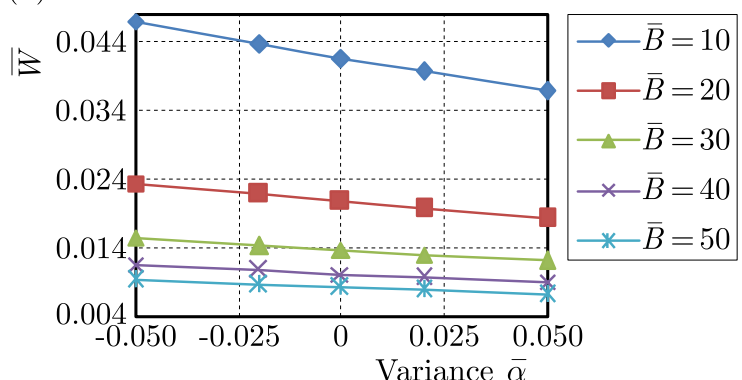

Fig. 6. Trends of $\bar{W}$ concerned with $\bar{\alpha}$ and: (a) $\bar{\varepsilon}$, (b) $\bar{B}$ 


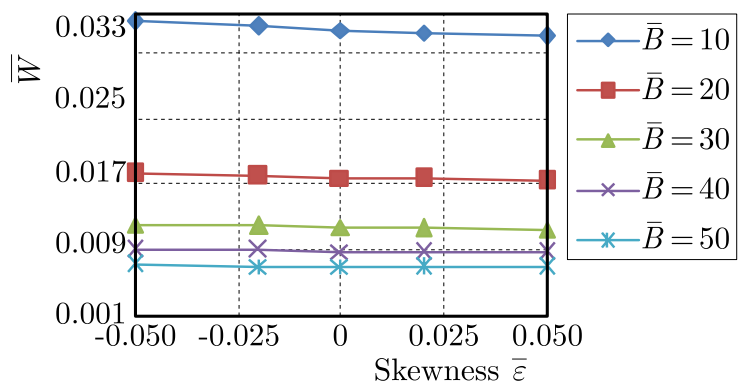

Fig. 7. Trends of $\bar{W}$ concern with $\bar{\varepsilon}$ and $\bar{B}$

\section{Validation}

The validation of the conclusion of this paper has been achieved by using the following comparison sets used in other publication. Tables 1 to 5 depicts the increase in the load carrying capacity over $14 \%$.

Table 1. Comparison of $\bar{W}$ calculated for $\bar{\alpha}$

\begin{tabular}{|c|c|c|c|}
\hline \multirow{2}{*}{ Quantity } & \multicolumn{3}{|c|}{ Load bearing capacity (calculated for } \\
& $\left.\mu^{*}=0.09, \bar{\sigma}=0.1, \bar{\varepsilon}=-0.05, \phi=0.1, \tau=0.1, m=0.3, \bar{B}=10, \bar{L}=0.5\right)$ \\
\hline $\bar{\alpha}$ & Result of the current study & Result of Patel and Deheri (2013a) & increase [\%] \\
\hline \hline-0.05 & 0.0148500 & 0.0115134 & 28.98 \\
\hline-0.02 & 0.0136554 & 0.0106446 & 28.28 \\
\hline 0 & 0.0129308 & 0.0101176 & 27.80 \\
\hline 0.02 & 0.0122575 & 0.0096279 & 27.31 \\
\hline 0.05 & 0.0113339 & 0.0089562 & 26.54 \\
\hline
\end{tabular}

Table 2. Comparison of $\bar{W}$ calculated for $\bar{\sigma}$

\begin{tabular}{|c|c|c|c|}
\hline \multirow{2}{*}{ Quantity } & \multicolumn{3}{|c|}{ Load bearing capacity (calculated for } \\
& $\left.\mu^{*}=0.09, \bar{\alpha}=0.02, \bar{\varepsilon}=-0.05, \phi=0.1, \tau=0.1, m=0.3, \bar{B}=10, \bar{L}=0.5\right)$ \\
\hline $\bar{\sigma}$ & Result of the current study & Result of Patel and Deheri (2013a) & increase [\%] \\
\hline \hline 0.1 & 0.0122575 & 0.0096279 & 27.31 \\
\hline 0.2 & 0.0114705 & 0.0090555 & 26.66 \\
\hline 0.3 & 0.0103649 & 0.0082515 & 25.61 \\
\hline 0.4 & 0.0091364 & 0.0073581 & 24.16 \\
\hline 0.5 & 0.0079318 & 0.0064819 & 22.36 \\
\hline
\end{tabular}

Table 3. Comparison of $\bar{W}$ calculated for $\bar{\varepsilon}$

\begin{tabular}{|c|c|c|c|}
\hline \multirow{2}{*}{ Quantity } & \multicolumn{3}{|c|}{ Load bearing capacity (calculated for } \\
& $\left.\mu^{*}=0.09, \bar{\sigma}=0.1, \bar{\alpha}=0.02, \phi=0.1, \tau=0.1, m=0.3, \bar{B}=10, \bar{L}=0.5\right)$ \\
\hline $\bar{\varepsilon}$ & Result of the current study & Result of Patel and Deheri (2013a) & increase [\%] \\
\hline \hline-0.05 & 0.0122575 & 0.0096279 & 27.31 \\
\hline-0.02 & 0.0120166 & 0.0094527 & 27.12 \\
\hline 0 & 0.0118614 & 0.0093399 & 26.99 \\
\hline 0.02 & 0.0117104 & 0.0092300 & 26.87 \\
\hline 0.05 & 0.0114912 & 0.0090706 & 26.68 \\
\hline
\end{tabular}


Table 4. Comparison of $\bar{W}$ calculated for $m$

\begin{tabular}{|c|c|c|c|}
\hline \multirow{2}{*}{ Quantity } & \multicolumn{3}{|c|}{ Load bearing capacity (calculated for } \\
& $\left.\mu^{*}=0.09, \bar{\sigma}=0.1, \bar{\varepsilon}=0.02, \phi=0.1, \tau=0.1, \bar{\alpha}=0.02, \bar{B}=10, \bar{L}=0.5\right)$ \\
\hline$m$ & Result of the current study & Result of Patel and Deheri (2013a) & increase [\%] \\
\hline \hline 0.1 & 0.0050043 & 0.0043529 & 14.96 \\
\hline 0.3 & 0.0117104 & 0.0092300 & 26.87 \\
\hline 0.5 & 0.0158152 & 0.0122153 & 29.46 \\
\hline 0.7 & 0.0184708 & 0.0141467 & 30.56 \\
\hline 0.9 & 0.0202714 & 0.0154562 & 31.15 \\
\hline
\end{tabular}

Table 5. Comparison of $\bar{W}$ calculated for $\bar{B}$

\begin{tabular}{|c|c|c|c|}
\hline \multirow{2}{*}{ Quantity } & \multicolumn{3}{|c|}{ Load bearing capacity (calculated for } \\
& $\left.\mu^{*}=0.09, \bar{\sigma}=0.1, \bar{\varepsilon}=0.02, \phi=0.1, \tau=0.1, m=0.3, \bar{\alpha}=0.02, \bar{L}=0.5\right)$ \\
\hline $\bar{B}$ & Result of the current study & Result of Patel and Deheri (2013a) & increase [\%] \\
\hline \hline 10 & 0.0117104 & 0.009230 & 26.87 \\
\hline 20 & 0.0058552 & 0.0046150 & 26.87 \\
\hline 30 & 0.0039035 & 0.0030767 & 26.87 \\
\hline 40 & 0.0029276 & 0.0023075 & 26.87 \\
\hline 50 & 0.0023421 & 0.0018460 & 26.87 \\
\hline
\end{tabular}

\section{Conclusion}

The impact of ferrofluid lubrication on the load bearing capacity of a short bearing system with a rough surface is studied. From the numerical computations performed, the analysis has yielded the following conclusions:

- Shliomis' ferrofluid flow provides relevant insights on the impact of rotations of the career liquid and magnetic particles. Furthermore, a varying magnetic field provides the benefit of creating the maximum field according to the necessary contact area of the bearing.

- This work is crucial because it provides more freedom than (Verma, 1986) and (Prajapati, 1994) regarding the magnitude.

- Standard deviation is the most important parameter in determining the performance of a bearing system of this type.

- Negatively skewed roughness aids the load carrying capacity and boosts the performance. Another thing to be understood is that a constant magnetic field shows a positive effect on the bearing capacity in the Shliomis model while the same is not true for the Neuringer-Rosensweig ferrofluid flow model.

- Furthermore, this article can create a new pathway for ensuring maximum utilization of the bearing system. It also clearly proposes that by managing the lubricant loss, the life span of the load bearing system can be increased substantially.

\section{Nomenclature}

$B \quad-\quad$ breadth of bearing

$g \quad$ - function of different parameters

$h, h_{m} \quad-$ film thickness and mean film thickness [mm]

$h_{s} \quad-$ deviation from mean level 


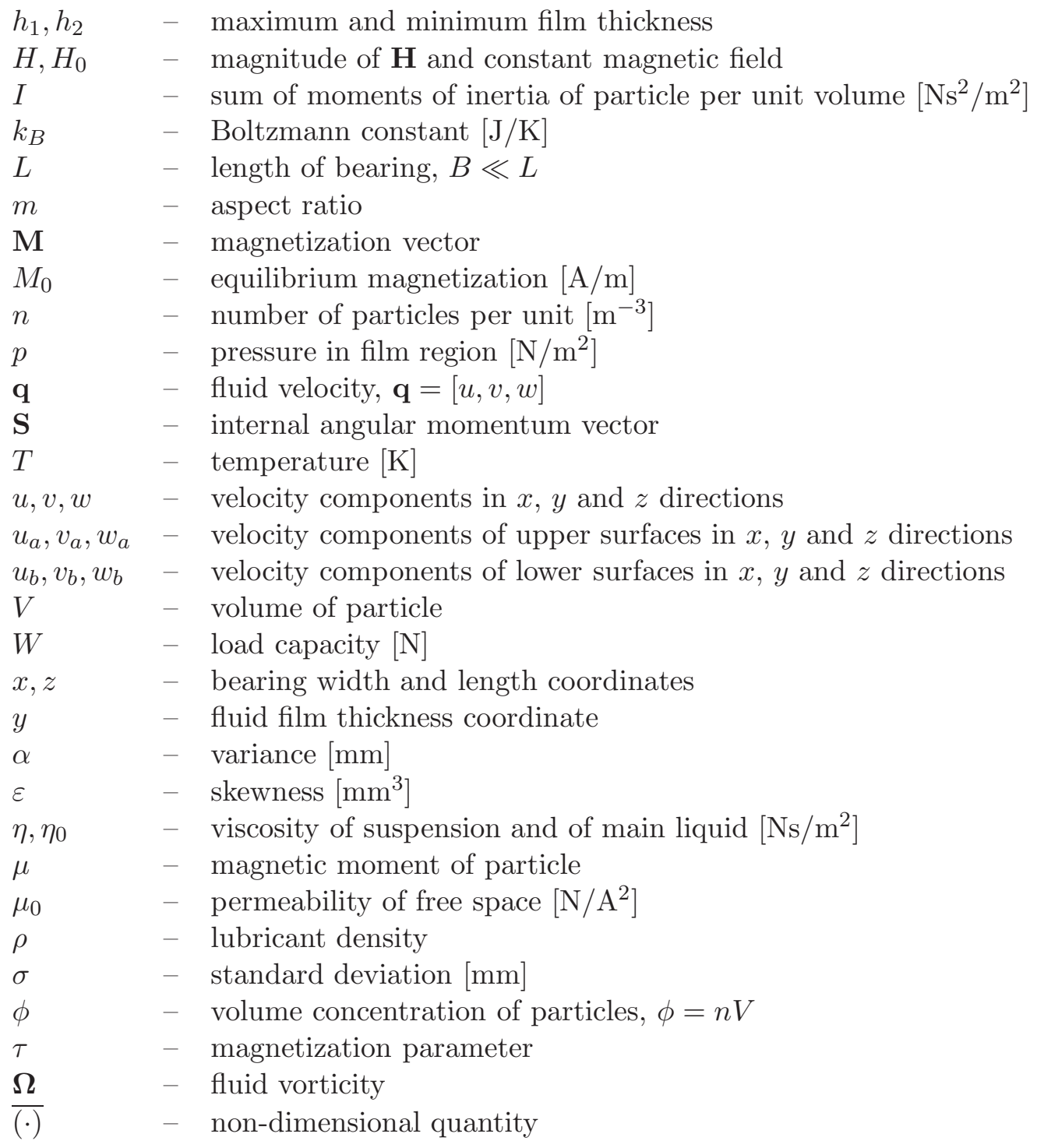

Acknowledgement

The authors acknowledge with regards the constructive comments, fruitful suggestions and remarks of the Reviewer/Editor, leading to an overhauling of the materials presented in the paper, necessitating the introduction of a few more references.

\section{References}

1. Basu S.K., Sengupta S.N., Ahuja B.B., 2005, Fundamentals of Tribology, Prentice-Hall of India Private Limited, New Delhi, India

2. Bнат M.V., 2003, Lubrication with a Magnetic Fluid, Team spirit Pvt. Ltd., India

3. Christensen H., Tonder K.C., 1969a, Tribology of rough surfaces: stochastic models of hydrodynamic lubrication, SINTEF Report No. 10/69-18

4. Christensen H., Tonder K.C., 1969b, Tribology of rough surfaces: parametric study and comparison of lubrication model, SINTEF Report, No. 22/69-18

5. Christensen H., Tonder K.C., 1970, The hydrodynamic lubrication of rough bearing surfaces of finite width, ASME-ASLE Lubrication Conference, Cincinnati, Ohio, USA 
6. Deheri G.M., Patel J.R., 2011, Effect of surface roughness on the performance of a magnetic fluid based short bearing, Mathematics Today, 27, 10-23

7. Lin J.R., Li P.J., Hung T.C., 2013, Lubrication performances of short journal bearings operating with non-Newtonian ferrofluids, Zeitschrift für Naturforschung, 68a, 249-254

8. Majumdar B.C., 2008, Introduction to Tribology of Bearings, S. Chand and Comp. Ltd., New Delhi, India

9. Patel J., Deheri G.M., 2013a, A comparison of porous structures on the performance of a magnetic fluid based rough short bearing, Tribology in Industry, 35, 3, 177-189

10. Patel R.U., Deheri G.M., 2013b, Effect of slip velocity on the performance of a short bearing lubricated with a magnetic fluid, Acta Polytechnica, 53, 6, 890-894

11. Patel R.M., Deheri G.M., Vadher P.A., 2010a, Magnetic fluid based short bearing and roughness effect, Journal of Science, 1, 1, 102-107

12. Patel R.M., Deheri G.M., Vadher P.A., 2010b, Performance of a magnetic fluid-based short bearing, Acta Polytechnica Hungarica, 7, 3, 63-78

13. Patel R.M., Deheri G.M., Vadher P.A., 2015, Hydromagnetic short bearings, Journal of Mechanical Engineering and Technology, 7, 2, 19-32

14. Patel N.S., Vakharia D.P., Deheri G.M., 2012, A study on the performance of a magnetic fluid based hydrodynamic short porous journal bearing, Journal of the Serbian Society for Computational Mechanics, 6, 2, 28-44

15. Prajapati B.L., 1994, Magnetic fluid-based porous inclined slider bearing with velocity slip, Prajna, 73-78

16. Shimpi M.E., Deheri G.M., 2010, Magnetic fluid based rough short bearing, Journal of the Balkan Tribological Association, 16, 4, 484-497

17. Shimpi M.E., Deheri G.M., 2012, Effect of deformation in magnetic fluid based transversely rough short bearing, Tribology - Materials Surfaces and Interfaces, 6, 1, 20-24

18. Shliomis M.I., 1972, Effective viscosity of magnetic suspensions, Soviet Physics - JETP, 34, 6, 1291-1294

19. Shliomis M.I., 1974, Magnetic fluids, Soviet Physics Uspekhi, 17, 2, 153-169

20. Verma P.D.S., 1986, Magnetic fluid-based squeeze films, International Journal of Engineering Sciences, 24, 3, 395-401 